\title{
Measurements of dijet azimuthal decorrelation at 8 TeV from CMS
}

\author{
Panagiotis KOKKAS*广 \\ University of Ioannina \\ E-mail: pkokkas@uoi.gr
}

\begin{abstract}
Measurements of the decorrelation of azimuthal angles between the two jets with the largest transverse momenta are presented for seven regions of leading jet transverse momentum. The analysis is based on data collected during 2012 with the CMS experiment, corresponding to an integrated luminosity of $19.7 \mathrm{fb}^{-1}$ of proton-proton collisions at $\sqrt{s}=8 \mathrm{TeV}$. The dijet azimuthal decorrelation is sensitive to the radiation of additional gluons and probes the dynamics of multijet production. The results are compared to fixed-order predictions of perturbative Quantum Chromodynamics, and to simulations using various Monte Carlo event generators that include parton showers, hadronization, and multiparton interactions.
\end{abstract}

XXIV International Workshop on Deep-Inelastic Scattering and Related Subjects

11-15 April, 2016

DESY Hamburg, Germany

\footnotetext{
* Speaker.

${ }^{\dagger}$ For the CMS Collaboration.
} 


\section{Introduction}

Quantum Chromodynamics (QCD) predicts the decorrelation in the azimuthal angle between the two leading in $p_{\mathrm{T}}$ jets, $\Delta \phi_{\text {dijet }}=\left|\phi_{\text {jet } 1}-\phi_{\text {jet2 } 2}\right|$, as a function of the number of jets produced. The two parton interaction at leading-order (LO) produces two final-state partons which are fully correlated in space and are produced in a back-to-back topology. Events with only two jets have small azimuthal decorrelations and $\Delta \phi_{\text {dijet }} \approx \pi$. As the number of jets increase in an event larger decorrelations, i.e. deviations from $\pi$, are observed. In this sense dijet azimuthal decorrelations is a useful tool to study QCD radiation effects over a wide range of jet multiplicities without the need to measure all the additional jets. Studies of the dijet azimuthal decorrelations are important because they provide an insight to the multi-partonic final states, which are still challenging in respect to the theoretical description by perturbative QCD (pQCD).

In these proceedings a measurement [1] of the dijet differential cross section as a function of the dijet azimuthal angular separation $\Delta \phi_{\text {dijet }}$, normalised by the dijet cross section integrated over the entire $\Delta \phi_{\text {dijet }}$ phase space,

$$
\frac{1}{\sigma_{\text {dijet }}} \frac{\mathrm{d} \sigma_{\text {dijet }}}{\mathrm{d} \Delta \phi_{\text {dijet }}},
$$

is presented. The measurement is performed for seven regions of the leading jet $p_{\mathrm{T}}, p_{\mathrm{T}}$ max , within a rapidity region of $|y|<2.5$. This observable, by normalising the $\Delta \phi_{\text {dijet }}$ distributions, minimises many experimental and theoretical uncertainties. For the first time, azimuthal angular separations $\Delta \phi_{\text {dijet }}$ over the full phase space from 0 to $\pi$ are covered. The measurement is compared to fixedorder predictions up to NLO for 3-jet production, and to NLO and LO dijet as well as to tree-level multijet production Monte Carlo event generators that include parton showers, hadronization, and multiparton interactions.

The measurement is performed using data collected during 2012 with the CMS [2] experiment at the CERN LHC, corresponding to an integrated luminosity of $19.7 \mathrm{fb}^{-1}$ of proton-proton collisions at $\sqrt{s}=8 \mathrm{TeV}$.

\section{Jet Reconstruction and Energy Calibrations at CMS}

CMS has developed the particle-flow (PF) event algorithm [3] which reconstructs and identifies each individual particle with an optimized combination of information from the various elements of the CMS detector. Jets are reconstructed by clustering the PF particle candidates with the collinear- and infrared- safe anti- $k_{\mathrm{T}}$ jet algorithm [ [ with a size parameter of $\mathrm{R}=0.7$ for this analysis.

Jet momentum is determined as the vectorial sum of all particle momenta in the jet, and (before corrections) is found from simulation to be within 5\% to $10 \%$ of the true momentum over the whole $p_{\mathrm{T}}$ spectrum and detector acceptance. The jet energy corrections (JEC) [5], 6] are calculated using a detailed Monte Carlo (MC) simulation of the detector, and are then adjusted for data using a combination of several channels and data-driven methods. The JEC successively correct for the offset energy coming from multiple proton-proton collisions in the same and adjacent beam crossings (pileup), the detector response to hadrons, and residual differences between data and MC 
simulation as a function of the jet pseudorapidity $\eta$ and transverse momentum $p_{\mathrm{T}}$. Corrections depending on jet flavor (for quarks: $\mathrm{u}$ and d, s, c and b; and for gluons), which are important for QCD studies are also applied. For a jet with a $p_{\mathrm{T}}$ of $100 \mathrm{GeV}$, the typical correction is about $10 \%$, and decreases with increasing $p_{\mathrm{T}}$. The consecutive steps of the JEC are illustrated in Fig. 1.

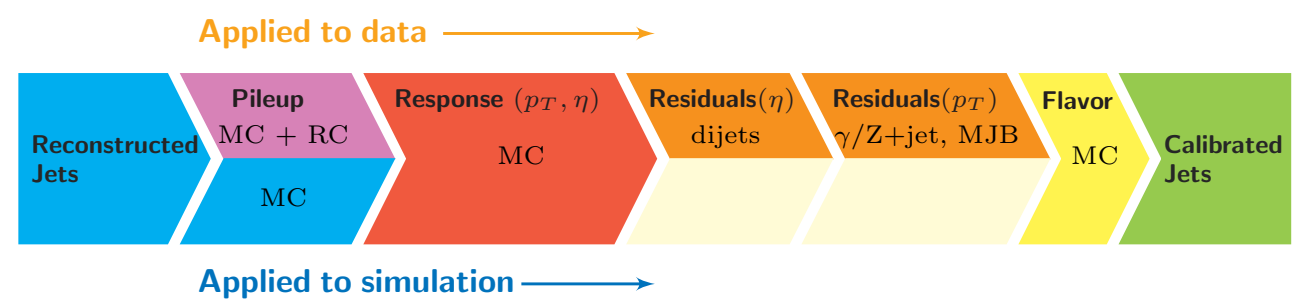

Figure 1: Consecutive stages of JEC, for data and MC simulation. All corrections marked with MC are derived from simulation studies, RC stands for random cone, and MJB refers to the analysis of multijet events.

Figure 1 summarizes the JEC systematic uncertainties as a function of jet $p_{\mathrm{T}}$ and for central jets. In the important parts of the phase space JEC uncertainties are of the order of $1 \%$, allowing thus CMS to perform precision physics with jets.

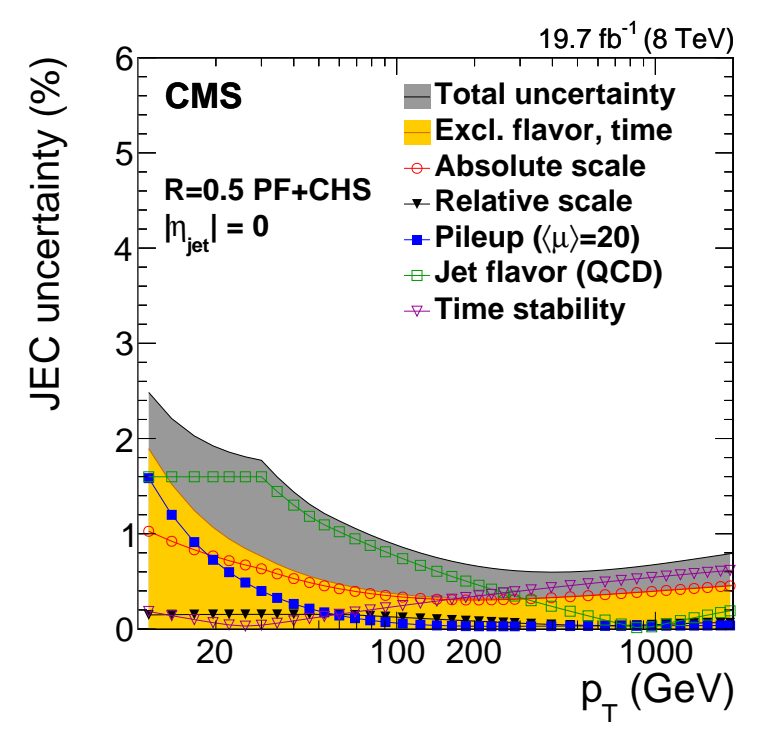

Figure 2: Summary of JES systematic uncertainties as a function of jet $p_{\mathrm{T}}$ for $\left|\eta_{\text {jet }}\right|=0$. The markers show the single effect of different sources, the gray dark band the cumulative total uncertainty. The total uncertainty, when excluding the effects of time dependence and flavor, is also shown in the yellow band.

\section{Measurements of dijet azimuthal decorrelation}

Figure 3 on the left shows the normalized dijet cross section differential in $\Delta \phi_{\text {dijet }}$ for seven $p_{\mathrm{T}} \max$ regions, scaled by multiplicative factors for presentation purposes. The $\Delta \phi_{\text {dijet }}$ distributions are strongly peaked at $\pi$ and become steeper with increasing $p_{\mathrm{T}} \max$. 
The results are compared to fixed-order predictions of perturbative QCD which are performed using the NLOJET++ program version 4.1.3 [7, 8] within the framework of the FASTNLO package version 2.3.1 [9]. The differential cross section is calculated for 3-jet production at NLO, with three or four partons in the final state. This calculation has LO precision in the region $\pi / 2 \leq \Delta \phi_{\text {dijet }}<$ $2 \pi / 3$ and NLO precision for $2 \pi / 3 \leq \Delta \phi_{\text {dijet }}<\pi$. In Fig. 目 on the left the predictions from LO (dashed line; $\pi / 2 \leq \Delta \phi_{\text {dijet }}<2 \pi / 3$ ) and NLO (solid line; $2 \pi / 3 \leq \Delta \phi_{\text {dijet }} \leq \pi$ ) calculations using the CT10 [10] NLO PDF set are overlaid to the data points. Around the theory lines, as hatched regions, it is shown the PDF, $\alpha_{S}$, and scale uncertainties added in quadrature to give the total theoretical uncertainty.

Figure 3 on the right shows the ratios of the normalized dijet cross section differential in $\Delta \phi_{\text {dijet }}$ to LO (triangles) and NLO (squares) pQCD predictions using the CT10 NLO PDF set for all $p_{\mathrm{T}}$ max regions. The error bars on the data points represent the total experimental uncertainty, while the uncertainties of the theoretical predictions are shown separately for PDF \& $\alpha_{S}$ and for scales. The theoretical predictions using other PDF sets relative to CT10 are also shown with different line styles.
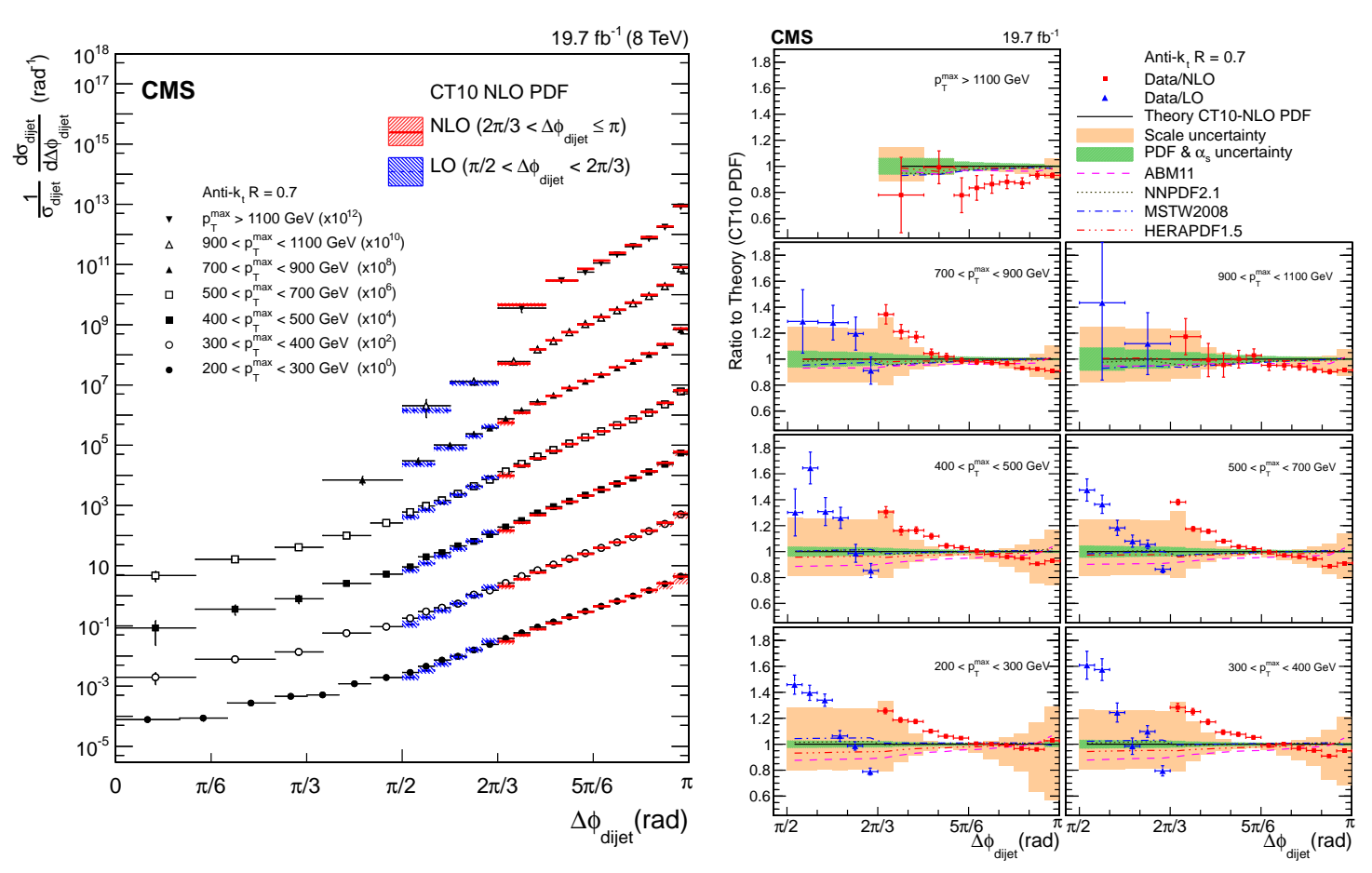

Figure 3: On the left, the normalized dijet cross section differential in $\Delta \phi_{\text {dijet }}$ for seven $p_{\mathrm{T}}$ max regions overlaid with predictions from LO (dashed line; $\pi / 2 \leq \Delta \phi_{\text {dijet }}<2 \pi / 3$ ) and NLO (solid line; $2 \pi / 3 \leq \Delta \phi_{\text {dijet }} \leq$ $\pi$ ) calculations using the CT10 NLO PDF set. On the right, the ratios to LO (triangles) and NLO (squares) pQCD predictions using the CT10 PDF set at next-to-leading evolution order for all $p_{\mathrm{T}}$ max regions.

A nice agreement between the fixed-order calculations and the data is observed for $\Delta \phi_{\text {dijet }}>$ $5 \pi / 6$, except for the highest $p_{\mathrm{T}}$ max region where the predictions exceed data. In the region $2 \pi / 3 \leq \Delta \phi_{\text {dijet }}<5 \pi / 6$ systematic discrepancies are observed which diminish with increasing $p_{\mathrm{T}} \max$. For $\Delta \phi_{\text {dijet }}<2 \pi / 3$ a similar pattern is observed but with less significance due to the 
larger scale uncertainties. Similar observations were made in previous CMS [11] and ATLAS [12] measurements.

The results are also compared to simulations using various Monte Carlo event generators that include parton showers, hadronization, and multiparton interactions. Figure 4 on the left shows the normalized dijet cross section differential in $\Delta \phi_{\text {dijet }}$ for seven $p_{\mathrm{T}}$ max regions, overlaid with predictions from the PYTHIA6 [13], HERWIG++ [14], PYTHIA8 [15], MADGRAPH [16] + PYTHIA6, and POWHEG [17, 18, 19] + PYTHIA8 event generators. The ratios of the event generators predictions to data are shown in the same figure on the right. The solid band indicates the total experimental uncertainty and the error bars on the MC points represent the statistical uncertainties of the simulated data.
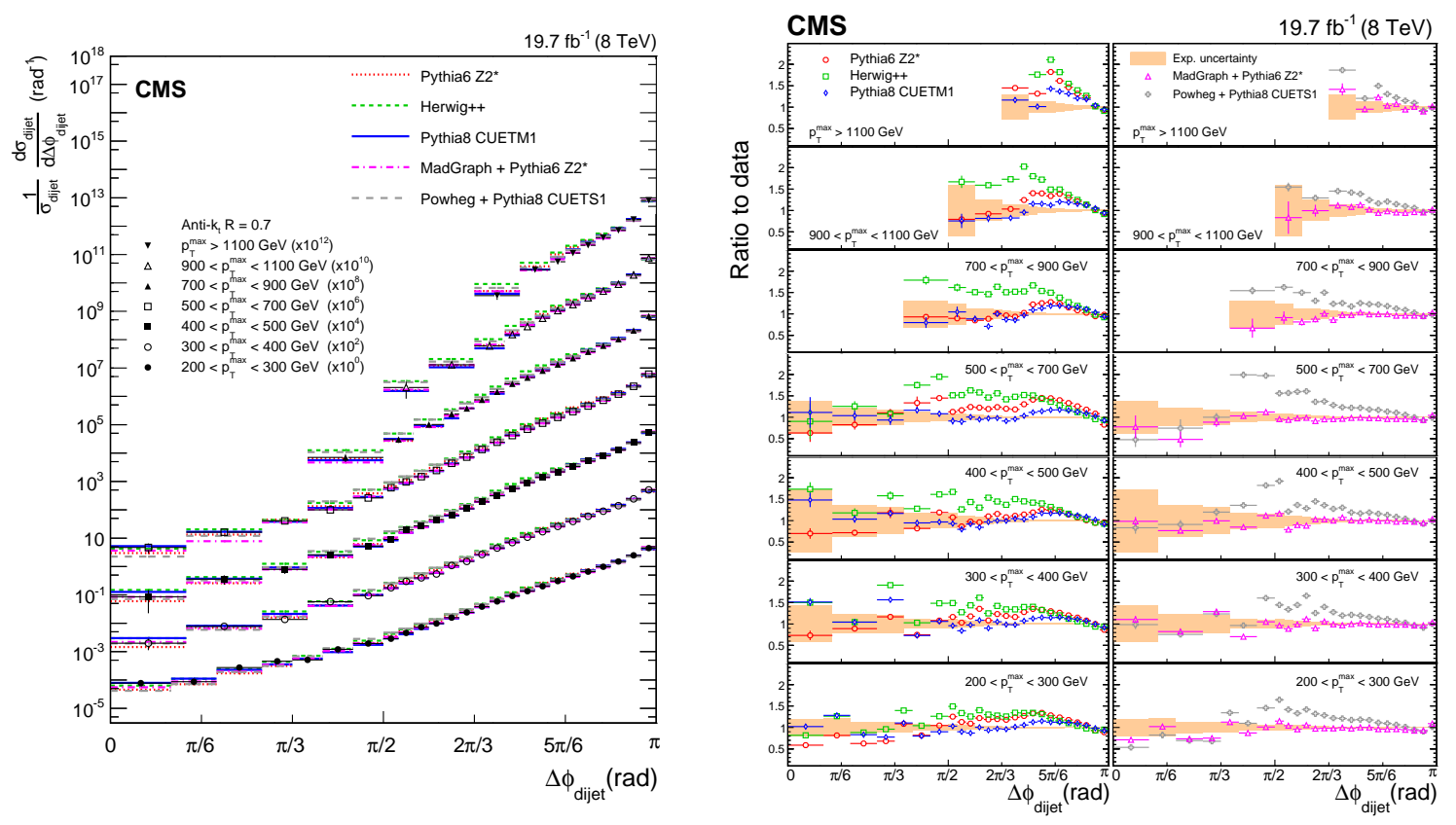

Figure 4: On the left the normalized dijet cross section differential in $\Delta \phi_{\text {dijet }}$ for seven $p_{\mathrm{T}} \max _{\text {regions. }}$ Overlaid on the data are predictions from the PYTHIA6, HERWIG++, PYTHIA8, MADGRAPH + PYTHIA6, and POWHEG + PYTHIA8 event generators. On the right the ratios of the event generators predictions to data.

The LO dijet event generators PYTHIA6, PYTHIA8, and HERWIG++ overshoot the data particularly around $\Delta \phi_{\text {dijet }}=5 \pi / 6$. Out of these three generators PYTHIA 8 exhibits the smallest deviations from the measurements. The tree-level multiparton event generator MADGRAPH interfaced with PYTHIA6 provides the best description of the measurement. Finally the POWHEG generator matched to PYTHIA8 employed only in the dijet NLO mode shows deviations from the data similar to the LO dijet event generators.

\section{Conclusions}

Measurements of the normalized dijet cross section differential in the azimuthal angular separation $\Delta \phi_{\text {dijet }}$ for seven regions in the leading-jet transverse momentum $p_{\mathrm{T}}{ }^{m a x}$, are presented. The measurements are performed using data collected during 2012 with the CMS experiment at the 
CERN LHC, corresponding to an integrated luminosity of $19.7 \mathrm{fb}^{-1}$ of proton-proton collisions at $\sqrt{s}=8 \mathrm{TeV}$.

The comparison of data to fixed-order NLO calculations in perturbative QCD for 3-jet production with up to four outgoing partons shows an overall agreement, although some systematic discrepancies are exhibited.

The results are also compared to simulations using various Monte Carlo event generators that include parton showers, hadronization, and multiparton interactions. Most of the generators exhibit deviations from the measurements. The best description of the measurement is given by the tree-level multiparton event generator MADGRAPH interfaced with PYTHIA6. The observations emphasize the need to improve predictions for multijet production.

\section{References}

[1] CMS Collaboration, arXiv:1602.04384 [hep-ex] (2016), submitted to EPJC.

[2] CMS Collaboration, JINST 3 (2008) S08004.

[3] CMS Collaboration, Physics Analysis Summary CMS-PAS-PFT-09-001.

[4] M. Cacciari, G. P. Salam, and G. Soyez, JHEP 04 (2008) 063.

[5] CMS Collaboration, .JINST 6 (2011) P11002.

[6] CMS Collaboration, Detector Performance Summary CMS-DP-2015-044.

[7] Z. Nagy, Phys. Rev. Lett. 88 (2002) 122003.

[8] Z. Nagy, Phys. Rev. D 68 (2003) 094002.

[9] D. Britzger, K. Rabbertz, F. Stober, and M. Wobisch, arXiv:1208.3641 [hep-ph] (2012).

[10] H.-L. Lai et al., Phys. Rev. D 82 (2010) 074024.

[11] CMS Collaboration, Phys. Rev. Lett. 106 (2011) 122003.

[12] ATLAS Collaboration, Phys. Rev. Lett. 106 (2011) 172002.

[13] T. Sjostrand, S. Mrenna, and P. Z. Skands, JHEP 05 (2006) 026.

[14] M. Bahr et al., EPJC 58 (2008) 639.

[15] T. Sjostrand, S. Mrenna, and P. Z. Skands, Comput. Phys. Commun. 178 (2008) 852.

[16] J. Alwall et al., JHEP 06 (2011) 128.

[17] S. Frixione, P. Nason, and C. Oleari JHEP 11 (2007) 070.

[18] S. Alioli, P. Nason, C. Oleari, and E. Re, JHEP 06 (2010) 043.

[19] P. Nason, JHEP 11 (2004) 040. 